\title{
Intramucosal adenocarcinoma arising within a colonic polypoid ganglioneuroma in a 21 year old female with Cowden syndrome
}

\author{
Anu Chittenden*, Joel E Goldberg, Jason L Hornick, Elena M Stoffel \\ From 13th Annual Meeting of the Collaborative Group of the Americas on Inherited Colorectal Cancer \\ Honolulu, Hawaii, USA. 16-17 October 2009
}

\begin{abstract}
Background
Cowden syndrome (part of the PTEN Hamartoma Tumor Syndromes) is a relatively rare autosomal dominant genodermatosis which is characterized by the growth of lesions in multiple organ systems, including skin, breast, thyroid, endometrium and central nervous system. While most of these lesions are benign, Cowden syndrome confers a substantial risk for several cancers including breast, thyroid and endometrial cancer. Hamartomas of the colon are seen; while there are reports of colon cancer in patients with Cowden syndrome, specialized screening for colon cancer is typically not recommended (NCCN Practice Guidelines in Oncology, v.l.2009). Here, we report a rare case of a large adenoma with intramucosal adenocarcinoma associated with a colonic ganglioneuroma in a young woman with confirmed Cowden syndrome.
\end{abstract}

\section{Case}

A 21 year old female presented to our colorectal surgery department for a second opinion regarding indications for surgical colectomy. Past history was remarkable for a right hemicolectomy at age 6 for an obstructing right colonic mass. At age 14, she underwent a colonoscopy that revealed multiple polyps which were ganglioneuromas. At age 18, she underwent genetic evaluation for Cowden syndrome and was found to have a deleterious mutation in the PTEN gene, R130Q (CGA->CAA). Physical exam was remarkable for macrocephaly, papillomatosis of the tongue and oral mucosa and several large lipomas on her back. Available family history was negative for Cowden-related diagnoses. At age 20, she developed chronic crampy abdominal pain, diarrhea, rectal bleeding and iron-deficiency anemia.

\footnotetext{
* Correspondence: achittenden@partners.org

Dana-Farber Cancer Institute and Brigham and Women's Hospital, Boston, Massachusetts 02115, USA
}

Colonoscopic examination revealed multiple polyps ranging in size from 0.3 to $1.2 \mathrm{~cm}$, which were prolapsing and causing intermittent obstruction. Biopsies of several polyps revealed ganglioneuromas, as well as fragments of tubular adenoma. She underwent subtotal colectomy with ileorectal anastomosis and examination of the excised colon revealed ganglioneuromatous polyposis ( $>100$ small polypoid ganglioneuromas with intramucosal ganglioneural proliferation in non-polypoid colonic mucosa) as well as four large $(2.5-6 \mathrm{~cm})$ ganglioneuromas. One of these large ganglioneuromas was associated with an adenoma with high-grade dysplasia and focal intramucosal adenocarcinoma. The patient did well after surgery, and the recommendation was made to continue endoscopic surveillance of the rectum every 6 months to screen for additional polyps.

\section{Conclusion}

Reports of malignant transformation of hamartomas are uncommon in Cowden syndrome. Interestingly, in one of the few reported cases of adenocarcinomas arising within hamartomas in Cowden syndrome, the position of the mutation (R130X) was the same as in this patient [1], indicating that there may be a genotype-phenotype correlation. This case illustrates the importance of endoscopic evaluation in Cowden patients with gastrointestinal complaints.

Published: 25 May 2010

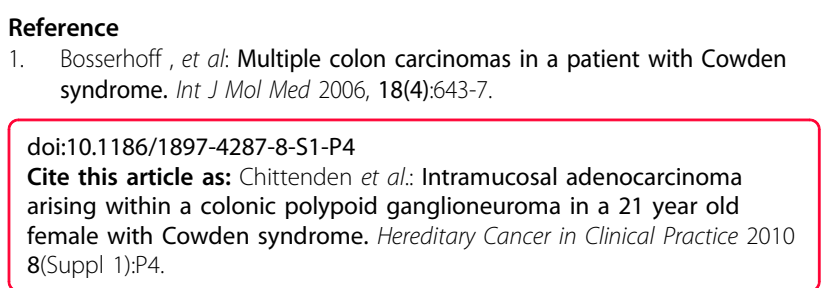

